Mecheril, Paul

\title{
Über die Kritik interkultureller Ansätze zu uneindeutigen Zugehörigkeiten
}

\section{- kunstpädagogische Perspektiven}

Clausen, Bernd [Hrsg.]: Teilhabe und Gerechtigkeit. Münster ; New York : Waxmann 2014, S. 11-19. (Musikpädagogische Forschung; 35)

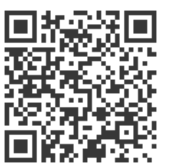

Quellenangabe/ Reference:

Mecheril, Paul: Über die Kritik interkultureller Ansätze zu uneindeutigen Zugehörigkeiten -

kunstpädagogische Perspektiven - In: Clausen, Bernd [Hrsg.]: Teilhabe und Gerechtigkeit. Münster ;

New York : Waxmann 2014, S. 11-19 - URN: urn:nbn:de:0111-pedocs-123441 - DOI: 10.25656/01:12344

https://nbn-resolving.org/urn:nbn:de:0111-pedocs-123441

https://doi.org/10.25656/01:12344

in Kooperation mit / in cooperation with:

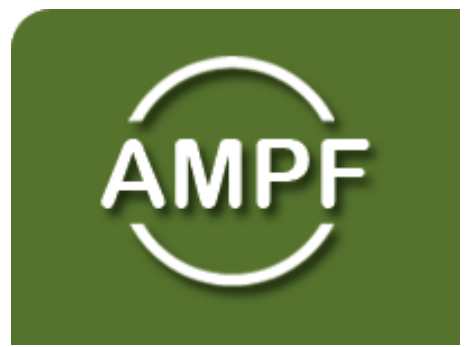

http://www.ampf.info

\section{Nutzungsbedingungen}

Gewährt wird ein nicht exklusives, nicht übertragbares, persönliches und beschränktes Recht auf Nutzung dieses Dokuments. Dieses Dokument ist ausschließlich für den persönlichen, nicht-kommerziellen Gebrauch bestimmt. Die Nutzung stellt keine Übertragung des Eigentumsrechts an diesem Dokument dar und gilt vorbehaltlich der folgenden Einschränkungen Auf sämtlichen Kopien dieses Dokuments müssen alle Urheberrechtshinweise und sonstigen Hinweise auf gesetzlichen Schutz beibehalten werden. Sie dürfen dieses Dokument nicht in irgendeiner Weise abändern, noch dürfen Sie dieses Dokument für öffentliche oder kommerzielle Zwecke vervielfältigen, öffentlich ausstellen, aufführen, vertreiben oder anderweitig nutzen.

Mit der Verwendung dieses Dokuments erkennen Sie die Nutzungsbedingungen an.

\section{Terms of use}

We grant a non-exclusive, non-transferable, individual and limited right to using this document.

This document is solely intended for your personal, non-commercial use. Use of this document does not include any transfer of property rights and it is conditional to the following limitations: All of the copies of this documents must retain all copyright information and other information regarding legal protection. You are not allowed to alter this document in any way, to copy it for public or commercial purposes, to exhibit the document in public, to perform, distribute or otherwise use the document in public.

By using this particular document, you accept the above-stated conditions of use.

\section{Kontakt / Contact:}

peDOCS

DIPF | Leibniz-Institut für Bildungsforschung und Bildungsinformation

Informationszentrum (IZ) Bildung

E-Mail: pedocs@dipf.de

Internet: www.pedocs.de

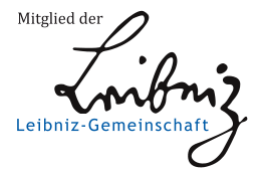




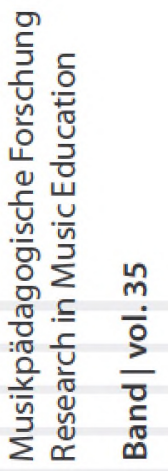

Bernd Clausen (Hrsg.)

\section{TEILHABE UND GERECHTIGKEIT PARTICIPATION AND EQUITY}

2
2
5
2
$x$
3
3 


\section{Musikpädagogische Forschung Research in Music Education}

Herausgegeben vom Arbeitskreis

Musikpädagogische Forschung e.V. (AMPF)

Band 35

Proceedings of the 35th Annual Conference of the German Association for Research in Music Education 
Bernd Clausen (Hrsg.)

Teilhabe und Gerechtigkeit Participation and Equity

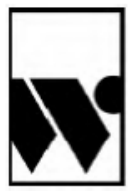

Waxmann 2014

Münster / New York 
Bibliografische Informationen der Deutschen Nationalbibliothek Die Deutsche Nationalbibliothek verzeichnet diese Publikation in der Deutschen Nationalbibliografie; detaillierte bibliografische Daten sind im Internet über http://dnb.d-nb.de abrufbar.

ISBN 978-3-8309-3144-7

ISSN 0937-3993

(c) Waxmann Verlag GmbH, Münster 2014

www.waxmann.com

info@waxmann.com

Umschlaggestaltung: Anne Breitenbach, Tübingen

Druck: Hubert \& Co., Göttingen

Gedruckt auf alterungsbeständigem Papier, säurefrei gemäß ISO 9706

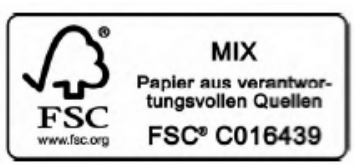

Printed in Germany

Alle Rechte vorbehalten. Nachdruck, auch auszugsweise, verboten.

Kein Teil dieses Werkes darf ohne schriftliche Genehmigung des Verlages in irgendeiner Form reproduziert oder unter Verwendung elektronischer Systeme verarbeitet, vervielfältigt oder verbreitet werden. 


\section{Inhalt}

Bernd Clausen

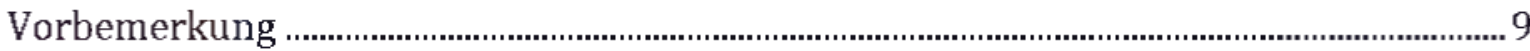

Editor's note

Paul Mecheril

Über die Kritik interkultureller Ansätze zu uneindeutigen Zugehörigkeiten kunstpädagogische Perspektiven .

On criticism of intercultural approaches towards amibiguous affiliations

Andreas Lehmann-Wermser \& Valerie Krupp

Musikalisches Involviertsein als Modell kultureller Teilhabe und Teilnahme

„Musical involvement": A theoretical model of cultural participation in music

Kerstin Heberle \& Ulrike Kranefeld

Zur Konstruktion von Leistungsdifferenz im instrumentalen Gruppenunterricht

Theoretische Perspektiven und forschungspraktische Überlegungen.

About the construction of difference in group instrumental lessons

Thomas Busch, Ulrike Kranefeld \& Svenja Koal

Klasseneffekte oder individuelle Einflussgrößen. Was bestimmt die Teilnahme am Instrumentallernen im Grundschulalter?

Class level or first level effects: What determines participation in instrumental learning during primary school?

Johannes Hasselhorn \& Andreas C. Lehmann

Entwicklung eines empirisch überprüfbaren Modells musikpraktischer

Kompetenz (KOPRA-M).

Developing an empirically testable model of practical music competency 
Ulrike Kranefeld, Kerstin Heberle, Birgit Lütje-Klose \& Thomas Busch

Herausforderung Inklusion? Ein mehrperspektivischer Blick auf die JeKi-Praxis an

Schulen mit gemeinsamem Unterricht (GU)

Inclusion Challenge? A Multiperspective View on 'JeKi' at Schools with Inclusive Schooling

Alexander J. Cvetko

Geschichten erzählen als Methode im Musikunterricht. Ergebnisse und

Forschungsmethoden einer historischen Studie

Storytelling as a teaching method in the music classroom. Results and methods of a historical study

Benedikt Ruf

Wie denken Lehrer*innen über (das Unterrichten von) Musiktheorie?

How do teachers think about (teaching) music theory?

Michael Rappe \& Christine Stöger

„Lernen nicht, aber ..." - Bildungsprozesse im Breaking

"Not Learning, But ...": Breaking and Educational Processes

Daniel Prantl

„Die Musikschule im Klassenzimmer". Streicherklassen aus der Perspektive von Prozess-Produkt-Didaktik.

"The Music School in the Classroom" - String Classes from the Viewpoint of ProcessProduct-Didactics

\section{Constanze Rora \& Cathleen Wiese}

,Verständige Musikpraxis' als Gegenstand von Musikunterricht zwischen Leiblichkeit und diskursivem Lernen.

The concept of 'Verständige Musikpraxis' as focus of music teaching between embodiment and discursive learning 
Jürg Zurmühle \& Isabelle Schmied

"Am Liebsten wollte ich nur noch zuhören, das konnte ich nicht, weil: ich musste singen." Eine Untersuchung zum Erleben von Kindern bei ihrer Teilnahme an einem Chorkonzert...

"I would just have liked to listen, but I couldn't because: I had to sing". Children's experience of participating in a choral performance

Daniel Mark Eberhard \& Rudolf-Dieter Kraemer

Augsburger Projekte und Initiativen zur Musikvermittlung. Versuch einer gründlichen Dokumentation

Augsburg Projects and Initiatives for Music Appreciation. Attempting a thorough documentation 
"Der Untersetzte: ,Der Paß ist der edelste Teil von einem Menschen. Er kommt auch nicht auf so einfache Weise zustand wie ein Mensch. Ein Mensch kann überall zustandkommen, auf die leichtsinnigste Art und ohne gescheiten Grund, aber ein Paß niemals. Dafür wird er auch anerkannt, wenn er gut ist, während ein Mensch noch so gut sein kann und doch nicht anerkannt wird.'

Ziffel: ,Aber Pässe gibt es hauptsächlich wegen der Ordnung. Sie ist in solchen [kriegerischen] Zeiten absolut notwendig. Nehmen wir an, Sie und ich liefen herum ohne Bescheinigung, wer wir sind, so daß man uns nicht finden kann, wenn wir abgeschoben werden sollen, das wär keine Ordnung. Sie haben vorhin von einem Chirurgen gesprochen. Die Chirurgie geht nur, weil der Chirurg weiß, wo zum Beispiel der Blinddarm sich aufhält im Körper. Wenn er ohne Wissen des Chirurgen wegziehn könnte, in den Kopf oder das Knie, würd die Entfernung Schwierigkeiten bereiten. Das wird Ihnen jeder Ordnungsfreund bestätigen." (B. Brecht: Flüchtlingsgespräche) (Brecht 2003, S. 7 f.).

Paul Mecheril

\section{Über die Kritik interkultureller Ansätze zu uneindeutigen Zugehörigkeiten - kunstpädagogische Perspektiven}

In den letzten Jahren ist in der Kunstpädagogik im amtlich deutschsprachigen Raum das Interesse an der Auseinandersetzung mit dem Themenfeld Migration deutlich gestiegen. Warum? Politisch und medial herrschte in Deutschland lange Zeit die Haltung vor, dass Migration randständig und nicht konstitutiv für hiesige gesellschaftliche Wirklichkeit sei. Bis Ende der 1990er Jahre war die offizielle Selbstdarstellung der Bundesrepublik hartnäckig dominiert von der Lebenslüge (Bade, 1994), Deutschland sei kein Einwanderungsland. Diese politische Irreführung und Ignoranz hat zweifelsohne die sozialen Folgen von Migration verkannt und zum Teil überaus problematische Entwicklungen nach sich gezogen, deren Konsequenzen sich deutlich auch im Feld der Bildung und Erziehung zeigen. In dieser gesamtgesellschaftlichen Ignoranz konnte auch die Kunstpädagogik sich nicht aufgefordert sehen, sich systematisch mit der Migrationstatsache auseinanderzusetzen, die nicht erst als Arbeits-

1 Der vorliegende Text greift auf Passagen bereits publizierter Texte zurück, vor allem Mecheril et al., 2008; Mecheril, 2009; Mecheril, 20012a. Der Beitrag erschien zuerst in Barbara LutzSterzenbach, Ansgar Schnurr, Ernst Wagner (Hg.), Bildwelten remixed. Transkultur, Globalität, Diversity in kunstpädagogischen Feldern. (2013). Bielefeld: transcript, S. 27-36. Für die Abdruckgenehmigung danken wir dem Autor und dem transcript-Verlag. 
migration nach 1945 einsetzte, sondern schon immer in unterschiedlicher Weise vorhanden war.

Seit Beginn des 21. Jahrhunderts hat sich das politische Szenario verändert und erste An-Erkennungen der Migrationstatsache setzen ein. Ab 2001 prägen bedeutsame Momente des Ortsansässigkeitsprinzips die deutsche Staatsbürgerschaftsregelung. Zudem wurde 2005 das Zuwanderungsgesetz verabschiedet, das zum ersten Mal den Begriff der Integration in einer migrationspolitischen Gesetzgebung verwendet. Zugleich macht bereits der Name des Gesetzes seine restringierende Ausrichtung deutlich: Gesetz zur Begrenzung und Steuerung von Zuwanderung. Bezogen auf Migration wird hier das widersprüchliche Prinzip politischer Regelung deutlich: Neuformierung des Sozialen durch Grenzausdehnung bei gleichzeitiger restriktiver Begrenzung.

Gleichwohl hat sich seit Beginn des neuen Jahrtausends in Deutschland das Selbstverständnis durchgesetzt, dass die Anwesenheit von Migrantinnen und Migranten weder marginal noch vorübergehend, sondern konstitutiv für gesellschaftliche Wirklichkeit ist. Die öffentlichen Diskurse, die politischen Debatten und Anstrengungen sowie die Ergebnisse der empirischen Bildungsforschung der letzten Jahre in Deutschland verweisen darauf, dass Migration zu den wichtigsten gesellschaftlichen Auseinandersetzungen der Gegenwart und Zukunft avanciert ist. Dies bestätigt auch das Feld, das sich aus pädagogischer Perspektive mit Kunst und Ästhetik befasst.

Wenn man mit einigem Risiko zu Pauschalisierung Regelmäßigkeiten in der „Entdeckung der Migrationstatsache in der Kunstpädagogik" benennen will, rücken mindestens drei Motive innerhalb des deutschsprachigen Diskurses in den Vordergrund:

- „Interkulturelle Kunstpädagogik“ als Weg der Integration derer, die einen s.g. Migrationshintergrund aufweisen

- „interkulturelle“ Öffnung von Bildungs- und Kulturinstitutionen

- "Interkulturelle Kunstpädagogik“ als Beitrag zu einem respektvollen und zivilen Umgang mit kultureller Differenz und Fremdheit

Somit sind die drei Schlüsselbegriffe des deutschsprachigen Diskurses über Migration auch in der Kunstpädagogik angekommen: „Integration“, „Mensch mit Migrationshintergrund“, die "kulturelle Differenz des Migranten“ (Mecheril, 2012a).

Ich möchte meinen Beitrag zu Fragen ästhetischer Bildung in der Migrationsgesellschaft mit einer kurzen Kritik der Besonderung durch die interkulturelle Perspekti$v e$ einleiten (ausführlicher Mecheril u.a., 2010), um vom Brett dieser Kritik in eine alternative Perspektive zu springen, die ich Migrationspädagogik nenne. Kritik meint selbstverständlich nicht die grundlegende Ablehnung von Überlegungen, Projekten, Texten oder Veranstaltungen, denen eines oder mehrere der drei Motive zugeordnet werden können. Ganz im Gegenteil finden sich innerhalb der pädagogischen Auseinandersetzung mit Kunst und Ästhetik wichtige und interessante Beiträge, selbst 
wenn sie auf Vokabeln zurückgreifen die das Bild bestätigen, Migranten und Migrantinnen seien "anders“.

Interkulturell ist ein Begriff, der darauf beharrt, dass die Anerkennung als gegeben verstandener kultureller Differenzen geboten sei. Anerkennung kultureller Differenz ist auch das zentrale Bildungsziel, das die Interkulturelle Pädagogik postuliert und anstrebt. Da wir auf Grund vielfältiger Bedingungen in kulturell pluralen und sich permanent wandelnden gesellschaftlichen Zusammenhängen leben, weist dieses Bildungsziel zwar vordergründig eine Plausibilität auf. Viele Zeitdiagnosen weisen jedoch darauf hin, dass moderne Gesellschaften als Zusammenhänge beschrieben werden müssen, für die aufgrund intensiver Dynamiken in zentralen gesellschaftlichen Bereichen und Sphären, nicht zuletzt auch durch weltweite Wanderungsbewegungen angestoßen, soziale Wandlungsprozesse, Diversifikation und Pluralisierungen charakteristisch sind. Diese Pluralisierung sozialer Kontexte, Stile, Selbstverständnisse und Beziehungen imponiert empirisch als Vielfalt kultureller Phänomene. Normativ verbindet sich hiermit häufig die Perspektive, dass diese Lebensformen in ihrer Differenz prinzipiell anzuerkennen seien. Die Ausbildung von Fertigkeiten wechselseitiger kommunikativer Anerkennung sei deshalb wertvoll und notwendig.

In einer kulturell pluralen (Welt-)Gesellschaft wird interkulturelles Lernen zu einer zentralen Bildungsaufgabe. Für die mittlerweile als eigenständiges pädagogisches Fachgebiet etablierte Interkulturelle Pädagogik ist der Bezug auf die interkulturelle Perspektive konstitutiv. Wer aber Texte zu Interkultureller Pädagogik liest, wird recht schnell feststellen, dass es sich bei der kulturellen Differenz, die im Rahmen Interkultureller Pädagogik thematisiert wird, nur um das Differenzverhältnis Migrant/in - Nichtmigrant/in handelt. Das Grundproblem der interkulturellen (Kunst-)Pädagogik besteht darin, dass sie eine spezifische Verschiedenheit immer schon voraussetzt. Diese (Voraus-)Setzung befördert zweierlei: Die Betonung des Kulturbegriffs suggeriert, dass „Kultur" die zentrale Differenzdimension sei, auf der migrationsgesellschaftliche Unterschiede zu beschreiben, zu untersuchen und zu behandeln seien. Dies schränkt aber Interkulturalität als Perspektive für gesellschaftliche, durch Migration hervorgebrachte Pluralität ein. Denn (migrations-)gesellschaftliche Differenzverhältnisse lassen sich nicht auf kulturelle Unterschiede reduzieren, da politische, ökonomische, rechtliche Dimensionen ebenso zu beachten sind. Solange sich Interkulturelle Pädagogik nicht mit der kulturellen Pluralität hoch differenzierter Gesellschaften in allgemeiner Einstellung beschäftigt, sondern im Wesentlichen sich mit Pluralisierung und Diversifizierung als Resultat von Migration auseinandersetzt, trägt „interkulturell“ zur kulturellen Besonderung von so genannten Menschen mit Migrationshintergrund (MmM), also zu der Erzeugung von Andersartigkeit und Fremdheit bei.

Der Ausdruck „kulturelle Differenz“ wird benutzt, um zwischen uns und jenen zu unterscheiden, die gewöhnlich als kulturell Andere imaginiert werden: die Fremden, die Zuwanderer, die Ausländerinnen, die Migrantinnen, die Menschen mit Migrationshintergrund etc. Und nur, weil es einen dominanten Diskurs gibt, in dem die Anderen (und nur sie und sie nur in dieser Weise) der kulturellen Differenz bezichtigt werden, 
kann über besondere Voraussetzungen und Erfordernisse nachgedacht werden, mit der Differenz zu den kulturell Anderen umzugehen. Da interkulturell mit Migranten verknüpft wird, können Sonderkompetenzen im Umgang mit Migrantinnen beispielsweise als interkulturelle Kompetenz entwickelt und nachgefragt werden. Die kulturelle Besonderung der MmM trägt komplementär dazu bei, dass die andere Seite - MoM - sich als nicht besonders, nicht integrationsbedürftig, normal und fraglos am richtigen Ort verstehen kann. In verwandter Weise hat Franz Hamburger (2009, S. 10) dies als das Elend der Interkulturellen Pädagogik bezeichnet:

„Es gibt unzählige Berichte über Besuche von Kindergartengruppen in Moschen und ausländischen Familien, aber keine Berichte über didaktisch analog konzipierte Besuche in Kirchen und deutschen Familien, um deren Kultur kennen zu lernen. Das ist immer noch das Elend der Interkulturellen Pädagogik“. Aber selbst, wenn man "deutsche Familien" besuchen würde, um ihre Kultur kennen zu lernen, wäre, da auch hier homogenisierende und pauschalisierende Zuschreibungen die Praxis strukturieren, das Elend der Interkulturellen Pädagogik nicht aufgehoben.

Im Hinblick auf das Anliegen der Interkulturellen Pädagogik schreibt Georg Auernheimer (2001, S. 45):

„Das Programm einer interkulturellen Bildung lässt sich auf zwei Grundprinzipien gründen: auf den Gleichheitsgrundsatz und den Grundsatz der Anerkennung anderer Identitätsentwürfe.“

Nun müsste man sich mit den Prinzipien, die Auernheimer hier anspricht, genauer auseinandersetzen. Ich will mich auf das zweite Prinzip, das der Anerkennung des und der Anderen und auch hierbei nur auf einen einzigen Punkt konzentrieren. Überspitzt formuliert lautet dieser: der Andere kann gar nicht anerkannt werden, da er als Anderer nicht erkennbar ist. Ein Erkennen setzt die Festschreibung (kultureller Andersheit) unabdingbar voraus. Das heißt nicht, dass ich Anerkennung für einen unangemessenen Grundsatz hielte, doch bedarf das Prinzip der Anerkennung einer Ergänzung: nämlich die Unmöglichkeit der Anerkennung. Sie bezeichnet die Einsicht, dass die Unmöglichkeit, etwas anzuerkennen, was nicht erkennbar ist, anerkannt werden sollte. Es geht mir hier um die Anerkennung der Nicht-Erkennbarkeit der Anderen, d.h. ihre Unbestimmtheit, in der die je eigene Unbestimmtheit einen Widerhall findet. Neben dem Gleichheitsgrundsatz und dem Prinzip der Anerkennung von Identitätsentwürfen, stellt das Paradoxon der Anerkennung der Unmöglichkeit von Anerkennung ein zentrales Moment allgemeiner Bildung in der Migrationsgesellschaft dar. 


\section{Ordnungen erkunden und uneindeutige Zugehörigkeiten (an)erkennen: Ästhetische Bildung in der Migrationsgesellschaft}

Wenn es im Fach Kunst und allgemein in der pädagogischen Auseinandersetzung mit Kunst und mit der ästhetischen Dimension des Lebens um „das Erlernen von Perspektiven der Wahrnehmung geht, die philosophische Fragen des Blickes und des Weltbildes einschließen" (Maset, 1995, S. 230), dann muss der Rückbezug auf die grundlegende Befragung des Blicks und des Weltbildes im Zusammenhang von Ästhetik und Migration, Kunstpädagogik und Migration den Ausgangspunkt darstellen. Im Rahmen der Perspektive Migrationspädagogik (Mecheril u.a. 2010) besteht die zentrale pädagogische Aufgabe für die Rahmung ästhetischer Bildungsprozesse darin, Situationen und Konstellationen zu arrangieren, in denen es für die Gegenüber (zum Beispiel Schülerinnen und Schüler) möglich wird, Schemata und Kategorien des Unterscheidens wahrzunehmen und zu reflektieren. Dies kann unter Nutzung vielfältiger symbolischer und ästhetischer Formen möglich werden, indem Verknüpfungen zwischen dem von ihnen rezeptiv und produktiv Wahrgenommenen zu vergangenen, gegenwärtigen und zukünftigen Zusammenhängen hergestellt sowie diese Assoziationen und Artikulationen gestaltet werden.

Es geht um das Erfahrbarwerden der Ästhetik von Zugehörigkeitsordnungen, verstanden als von politisch-sinnlich-kulturellen Kontexten vermittelte Dimension. Zugehörigkeitsordnungen haben dabei sozialisierende oder besser: subjektivierende Wirkung. Sie vermitteln Selbst-, Fremd- und Weltverständnisse nicht nur kognitiv, sondern vor allem auch sinnlich-leiblich. In diesen Verständnissen spiegeln sich soziale Positionen und Lagerungen sowie die differentielle Verteilung von materiellen und symbolischen Gütern und Anrechten. An diesem Punkt heißt Wahrnehmungswahrnehmung, sich zu den eigenen Wahrnehmungsschemata in ein (sinnliches) Verhältnis zu setzen. Es geht hier also nicht um Projekte ästhetischer Bildung, die durch das Machen und Hören von Musik, das Machen und Sehen von Theaterstücken, das Machen und Anfassen von Plastiken und Skulpturen, durch Erkundungen eigener und fremder Räume, Praxen und Geschichten zu mehr Toleranz, zu mehr Freundlichkeit und Achtsamkeit im Umgang mit dem Fremden und Anderen beitragen wollen (das interkulturelle Paradigma). Vielmehr stehen die verschiebende Erkundung des Schemas, das zwischen denen und diesen unterscheidet und seine sinnlich-leibliche Verankerung im Zentrum einer migrationspädagogisch informierten ästhetischen Bildung. Es geht hierbei darum, einen ästhetischen Rahmen zu schaffen, in dem Lernende und Lehrende mit Hilfe des Gestaltens (qua) symbolischer Formen Ordnungen und die eigene Position innerhalb dieser Ordnungen nicht nur kennenlernen, sondern auch ausprobieren, anprobieren, verändern und verwerfen.

In den letzten Jahren hat sich eine bedeutsame kulturwissenschaftliche Erkenntnisperspektive entwickelt: Dualistische Sichtweisen auf Kultur, Differenz und Identi- 
tät sollen aufgeschlossen und geöffnet werden. Die Perspektive operiert, wenn wir sie politisch wenden, mit einer doppelten Maxime: Identitäts- und Differenzkonzepte sollen so erweitert und modifiziert werden, dass nicht allein starre, kontextfreie, schattierungsarme, binäre und eindeutige Identitäts- und Differenzverhältnisse theoretisch-begrifflich gefasst werden. Sondern es geht darum, Phänomene der Uneindeutigkeit, des Changierens, des Übergangs praktisch anzuerkennen. Der differenztheoretische Diskurs hat sich analytisch-deskriptiv wie auch normativ-präskriptiv den Zwischentönen, Randgängen und Überschreitungen zugewandt.

Theoriediskurse, die sich um Kategorien wie Ambivalenz (Bauman, 1995), Dekonstruktion (Butler, 1991), Transdifferenz (Lösch, 2005) oder Unreinheit (Mecheril, 2009) gruppieren, markieren in jüngeren Debatten eine Verschiebung des Fokus. Es geht hier nicht nur darum, die Ordnung, Teilung, Grenzziehung und Grenze konstituierenden Momente zu untersuchen. Vielmehr werden der Ordnung entgegenlaufende Prozesse, Phänomene der Verunreinigung, Entgrenzung, der Verschiebung und Versetzung, der Neumischung und des Remix (siehe die Vorschläge der Nürnberger Kunstpädagogikkonferenz) in den Blick genommen.

Die Auffassung, dass Differenz die Scheidelinie binär organisierter Identitätskategorien darstelle, ist im Zuge dieser Theoriediskurse nachhaltig ins Wanken geraten. Ein Verständnis von Differenz als Ausdruck und Repräsentation einer benennbaren Trennung zwischen vermeintlichen Antagonismen suggeriert, dass das, was als Unterschiedenes trennt und verbindet, erfassbar sei. Es gehört aber zum Wesen des zu Unterscheidenden, zum Wesen der Relationierung, dass es „wesenlos“ ist. In diesem Zuge wird Differenz nicht als „bloßer" Unterschied, als das von einem identifizierbaren Eigenen klar abgegrenzte Andere verstanden. Vielmehr werden Gegensätzlichkeiten - Eigenes und Anderes - als in einer unauflöslichen Beziehung stehend begriffen, die die Identifizierbarkeit der antagonistischen Pole grundlegend problematisiert. Gleichzeitig wird versucht, der Unreinheit, der Unrepräsentierbarkeit und der Prozesshaftigkeit von Differenz-Phänomenen Rechnung zu tragen. Mit der Anerkennung der Verwobenheit von diverser, sich prozesshaft verändernder Differenz und Identität wird die „Entweder-oder“-Ordnung fraglich. Diese Zusammenhänge verdichten sich zuweilen im Begriff Hybridität.

Hybridität weist nun zumindest zwei wesentliche Dimensionen auf, die für kunstpädagogische Perspektiven relevant sein können. In der ersten Dimension meint Hybridität eine technologische Leistungssuggestion und eine medizinische Heilsofferte, weil in dem Ausdruck Hybridität das nicht-funktionale Beharren auf der Reinheit verwandter Materialien, Programme und Systeme leistungssteigernd überwunden zu werden scheint. In diesem technisch-medizinischen Sektor steht Hybridität für Intelligenzsteigerung durch kalkulierte Unreinheit.

In anderen Sektoren, der eher auf ein junges Publikum gerichteten ästhetischen Industrie, eröffnet Hybridität ein anderes Spiel mit Differenz. Hier - auf den Märkten der Videoclips und Websites - wird Hybridität als Identitäts- und Beziehungsform gefeiert, als performatives Darstellungsbild und Inszenierungsmodus. Hier ist hybrid ein positiv besetzter Terminus im globalen Kontext, in dem nicht nur kulturelle Sy- 
nergien genutzt werden, sondern die betörende Inszenierung der Differenz als Code eingesetzt wird. Waren werden mit Bedeutung und Bedeutungen mit Produkten versehen, so dass in den Anrufen, Ansingungen und Angeboten dieser WareBedeutungskomplexen aus Individuen Subjekte werden.

Die Konjunktur des Wortes Hybridität tendiert dazu, nicht genauer hinzusehen, wo und wie Hybridität zu einer Disziplinierungspraxis wird. Wer etwa nicht gelernt hat, seine kulturelle Herkunft zumindest performativ und dem Augenschein nach zu transzendieren, wer provinziell geblieben ist, der und die bleibt im kapitalen Globalismus synkretistisch symbolisierter Zugänge zu Markt und Menschen auf der Strecke. Es macht also Sinn, von dem Feiern der „Hybridität“ zurück zu treten und die Praxis "Hybridität" auf die mit ihr verbundenen Ausschlüsse und Bemächtigungen zu betrachten. Ein Ausschluss-/Bemächtigungstyp heißt also: Hybridisierungen sind ein Disziplinierungs- und Leistungssteigerungsmittel des gegenwärtigen Kapitalismus.

Komplementär dazu positioniert sich eine weitere Dimension von Hybridität bzw. ein Hybriditätsverständnis, wie es im Bereich der postcolonial studies (vgl. etwa Castro Varela \& Dhawan, 2005) anzutreffen ist. Hybridität und hybrides Handlungsvermögen stellen Phänomene der Überschreitung und Zurückweisung binärer Unterscheidungen dar, die in zweierlei Hinsicht widerständig sein können. Zum einen widersetzt sich Hybridität dem universellen Anspruch binär unterscheidender Schemata, sie verweigert sich der allein oppositionellen Repräsentation und Konstruktion sozialer Prozesse und Antagonismen:

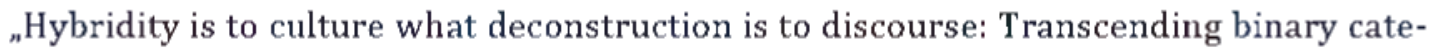
gories" (Nederveen Pieterse, 1998, S. 238).

Es muss, nun mit Rückbezug auf die erste Dimension, darum gehen empirisch genauer zu fragen, wer in der Lage ist, solche hybriden, wiederständigen Prozesse zu gestalten, also für wen klare Unterscheidungen von Wir und Nicht-Wir, von eigener und fremder Kultur ihre Verbindlichkeit verlieren (können) und wer mit der Unüberwindbarkeit dieser Unterscheidungen konfrontiert ist.

Dies kann auch mit Bezug auf das Nürnberg-Papier (in diesem Band2) gefragt werden. Hin und wieder gerät es in das Fahrwasser einer Argumentation intellektualistischer Voreingenommenheit, die den Diskurs um „hybrid“ und „transkulturell“ kennzeichnet (Mecheril, 2012b). Ein Ziel einer Kunstpädagogik im dritten Jahrtausend könnte darin bestehen, eine Achtsamkeit dafür zu pflegen, wo (alltagsweltlich) das Bewusstsein und das Leiden daran, dass es manchen zugestanden ist, die Unterscheidung zwischen „innen" und „außen“, von „eigen“ und „fremd“ zu transzendieren und anderen nicht, eine ästhetische und politische Form findet, die zu weniger machtvollen Verhältnissen beiträgt.

Es geht also vor dem Hintergrund dieser Grundspannung des Hybriden nicht so sehr um die Frage, welche Kultur, welche Bilder, welche besonderen ästhetischen Verhaltensweisen spezifische Migrantengruppen haben, wie diese Kultur zu be-

2 Siehe Fußnote 1. 
schreiben ist und wie unter den unterschiedlichen kulturellen Gruppen Verständigung möglich ist usw. Vielmehr geht es um die Frage, aufgrund welcher kulturellen Praktiken in pädagogischen Zusammenhängen zwischen „Migrantinnen“ und „NichtMigrantinnen" unterschieden wird, auf Grund welcher Bedingungen und in welchen Bildern "Migrantinnen“ als Migrantinnen wahrgenommen werden. Es geht ferner darum, wie Kinder lernen können, sich als Nicht-Ausländerin oder Nicht-Fremde zu verstehen und wie in alltäglichen Praxen innerhalb und außerhalb der offiziellen Orte neue, widerständige Formen der Überschreitung der traditionellen Grenzen erprobt und eingeübt werden können, eine Erkundung also der Praxen, Lebensweisen und Geschichten, die sich dem eindeutigen Unterscheiden entziehen.

Achtsamkeit für dieses alltagsweltlich kreative Potenzial von wandernden, nicht eindeutigen Positionen und Praxen, ist m.E. einer der zentralen Bezugspunkte migrationspädagogisch informierter ästhetischer Bildung.

\section{Literatur}

Auernheimer, G. (2001). Anforderungen an das Bildungssystem und die Schulen in der Einwanderungsgesellschaft. In G. Auernheimer (Hrsg.), Migration als Herausforderung für pädagogische Institutionen (S. 45-58). Opladen: Leske + Budrich.

Bade, K. (1994). Homo Migrans - Wanderungen von und nach Deutschland. Erfahrungen und Fragen. Essen: Klartext Verlag.

Bauman, Z. (1995). Moderne und Ambivalenz. Frankfurt a. M.: Fischer.

Brecht, B. (2003). Flüchtlingsgespräche. Frankfurt a. M.: Suhrkamp.

Butler, J. (1991). Das Unbehagen der Geschlechter. Frankfurt a. M.: Suhrkamp.

Castro Varela, M. \& Dhawan N. (2005). Postkoloniale Theorie. Eine kritische Einführung. Bielefeld: transcript.

Hamburger, F. (2009). Abschied von der Interkulturellen Pädagogik. Plädoyer für einen Wandel sozialpädagogischer Konzepte. Weinheim: Juventa.

Lösch, K. (2005). Begriff und Phänomen der Transdifferenz: Zur Infragestellung binärer Differenzkonstrukte. In L. Allolio-Näcke, B. Kalscheuer \& A. Manzeschke (Hrsg.), Differenzen anders denken. Bausteine einer Kulturtheorie der Transdifferenz (S. 22-45). Frankfurt a. M.: Campus.

Maset, P. (1995). Ästhetische Bildung und Differenz. Kunst und Pädagogik im technischen Zeitalter. Stuttgart: Radius.

Mecheril, P. (2009). Hybridität, kulturelle Differenz und Zugehörigkeiten als pädagogische Herausforderung. In G. Mertens u. a. (Hrsg.), Handbuch der Erziehungswissenschaft (Teilband: Umwelten; hg. von Ch. Alleman-Ghionda). Bonn: GörresGesellschaft.

Mecheril, P. (2012a). Ästhetische Bildung und Kunstvermittlung. Migrationspädagogische Anmerkungen. In Institut für Auslandsbeziehungen (ifa), Institute for Art Education (IAE), Zürcher Hochschule der Künste ZHdK Institut für Kunst im Kontext der Universität der Künste Berlin (Hrsg.), Kunstvermittlung in der Migrationsgesellschaft/ Reflexionen einer Arbeitstagung - 2011 (S. 26-35). Stuttgart: ifa. 
Mecheril, P. (2012b). Kritik der Hybridität. Kommentar zum Nürnberg-Paper. In Brenne, A., Sabisch, A. \& Schnurr, A. (Hrsg.), revisit. Kunstpädagogische Handlungsfelder \#teilhaben \#kooperieren \#transformieren. Schriftenreihe Kunst Pädagogik Partizipation. Buch 02 (S. 231-232). München: kopaed.

Mecheril, P., Castro Varela, M., Dirim, I., Kalpaka, A. \& Melter, C. (2010). BACHELOR | MASTER: Migrationspädagogik. Beltz: Weinheim.

Mecheril, P., Probadnick, D. \& Scherschel, K. (2008). (De)Binarisierung und Bildung. Empirisch-theoretische Vignetten eines Zusammenhangs. In L. Allolio-Näcke \& B. Kalscheuer (Hrsg.), Kulturelle Differenzen begreifen. Das Konzept der Transdifferenz aus interdisziplinärer Sicht (S. 383-406). Frankfurt a. M.: Campus.

Nederveen Pieterse, J.P. (1998). Der Melange-Effekt. In U. Beck (Hrsg.), Perspektiven der Weltgesellschaft (S. 87-124). Frankfurt a. M.: Suhrkamp.

Paul Mecheril

Carl-von-Ossietzky-Universität

Fakultät I

Ammerländer Heerstraße 114-118

D-26129 oldenburg

paul.mecheril@uni-oldenburg.de 